\title{
SPE-8, a protein-tyrosine kinase, localizes to the spermatid cell membrane through interaction with other members of the SPE-8 group spermatid activation signaling pathway in C. elegans
}

Paul J Muhlrad ${ }^{1,2}$, Jessica N Clark ${ }^{3}$, Ubaydah Nasri ${ }^{3}$, Nicholas G Sullivan ${ }^{3}$ and Craig W LaMunyon ${ }^{3 *}$

\begin{abstract}
Background: The SPE-8 group gene products transduce the signal for spermatid activation initiated by extracellular zinc in C. elegans. Mutations in the spe-8 group genes result in hermaphrodite-derived spermatids that cannot activate to crawling spermatozoa, although spermatids from mutant males activate through a pathway induced by extracellular TRY-5 protease present in male seminal fluid.

Results: Here, we identify SPE-8 as a member of a large family of sperm-expressed non-receptor-like protein-tyrosine kinases. A rescuing SPE-8::GFP translational fusion reporter localizes to the plasma membrane in all spermatogenic cells from the primary spermatocyte stage through spermatids. Once spermatids become activated to spermatozoa, the reporter moves from the plasma membrane to the cytoplasm. Mutations in the spe-8 group genes spe-12, spe-19, and spe-27 disrupt localization of the reporter to the plasma membrane, while localization appears near normal in a spe-29 mutant background.

Conclusions: These results suggest that the SPE-8 group proteins form a functional complex localized at the plasma membrane, and that SPE-8 is correctly positioned only when all members of the SPE-8 group are present, with the possible exception of SPE-29. Further, SPE-8 is released from the membrane when the activation signal is transduced into the spermatid.
\end{abstract}

Keywords: Caenorhabditis elegans, Spermatogenesis, Sperm activation, spe-8, Signal transduction

\section{Background}

The timing of sperm activation requires precise regulation. Activating too early wastes stored resources, and delayed activation put the sperm at a competitive disadvantage. Sperm activation, and spermatogenesis as a whole, have emerged as an important model system in the hermaphroditic nematode C. elegans [1]. Activation, also called spermiogenesis in C. elegans, is the final developmental transition in spermatogenesis when quiescent spherical spermatids undergo wholesale cellular reorganization, extend pseudopods, and begin to crawl. Two signaling molecules induce spermiogenesis:

\footnotetext{
*Correspondence: cwlamunyon@csupomona.edu

${ }^{3}$ Department of Biological Science, California State Polytechnic University, 3801 W. Temple Ave, Pomona, CA 91768, USA

Full list of author information is available at the end of the article
}

extracellular zinc [2] and the extracellular protease TRY-5 [3]. Both pathways are employed for male sperm activation, but only the zinc pathway is utilized in hermaphrodites for self-sperm activation [2]. The zinc signal is transduced into the spermatid via the products of at least five genes (spe-8, spe-12, spe-19, spe-27, and spe-29) named the spe- 8 pathway for the first of the genes discovered [4]. Loss-of-function mutations in any of these genes result in an identical phenotype: hermaphrodites are self-sterile because their spermatids do not activate, while male mutants are fertile. Mutant hermaphrodites become self-fertile when inseminated by males because their self-spermatids are activated by TRY- 5 in the seminal fluid. Given that spe-12, spe-19, and spe-29 encode membrane proteins [5-7], it has been proposed that a complex of the SPE- 8 group proteins 
forms at the cell membrane to transduce the zincderived signal into the cell. Although spe-8 was the first gene of this group to be named, the molecular identity of spe- 8 has not been published. Here, we report the identification of the spe- 8 coding sequence, show that it encodes a member of a large family of sperm-expressed protein tyrosine kinases, and demonstrate that its gene product localizes to the membrane in a manner dependent upon the presence of the other spe-8 group gene products.

\section{Results}

Identification of the spe- 8 coding sequence

Previous mapping placed spe- 8 to the left end of Chromosome I, approximately $8.7 \mathrm{cM}$ to the left of lin-17 [4]. Subsequently, spe-8 was positioned extremely close (inseparable) to mex-3 [8] (Figure 1A). Because essentially all spe and fer genes in C. elegans exhibit sperm-specific expression $[9,10]$, we reasoned that spe-8 expression is also specific to spermatogenesis. We surveyed microarray data comparing transcript abundance

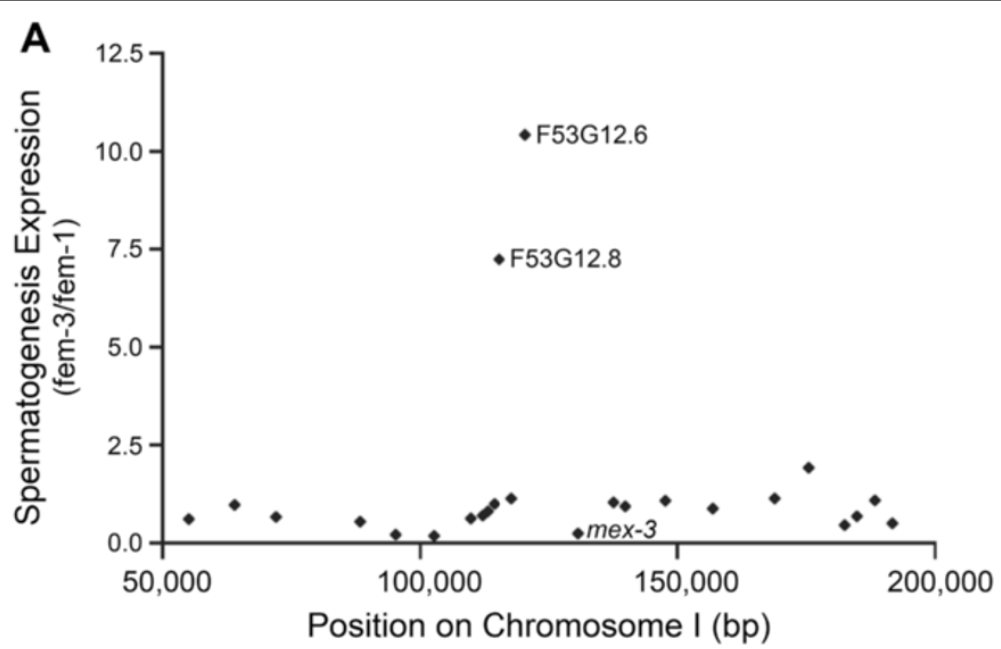

B
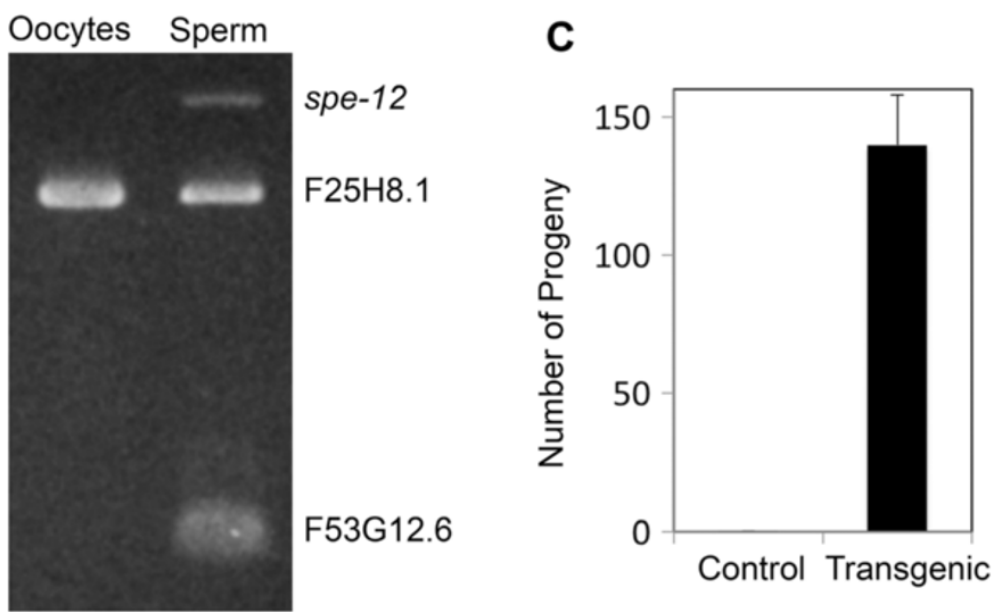

Figure 1 Identification of the spe- 8 coding sequence. A. The graph shows all the predicted genes on the far left end of Chromosome I, where spe- 8 and mex-3 are known to be very close to one another. The $Y$ axis plots the degree of sperm-specific expression measured as fem-3 (q23)/fem-I(hCl7) microarray signal intensity [9,10]. Two genes in the region, F53Gl2.6 and F53GI2.8, appear spermatogenesis-specific. F53GI2.8 had been previously eliminated as a candidate based on other results (Jeremy Nance, personal communication). B. Differential PCR analysis was used to confirn the sperm-specific expression of F53G12.6. PCR products were amplified from cDNA libraries derived either from fem-1(hcl7ts) hermaphrodites which produce only oocytes, or from fem-3(q23ts) hermaphrodites which produce only sperm. The PCR reactions were multiplexed for three possible products: (i) a 169 bp product from F53G12.6 CDNA, (ii) a 526 bp product from F25H8.1, a somatic gene included as a non-germline-specific control, and (iii) a 714 bp product from spe-12, included as a sperm-specific control. Both F53G12.6 and spe-12 products appear sperm specific by their appearance only in the fem-3 library, whereas the product from the somatic gene F25H8.1 was found in both libraries as expected. (PCR primers are given in Additional file 1). C. The results of transformation-rescue of spe-8(hc50) with wild-type PCR product containing F53G12.6 and its flanking sequence. Transformant worms produced a mean of 139.8 progeny $(n=12$; SEM $=18.0)$ compared to a mean of 0.1 progeny $(n=15 ; S E M=0.1)$ produced by their non-transformed siblings, indicating that F53G12.6 is spe-8. 
in hermaphrodites making only sperm [fem-3(q23gf)] with hermaphrodites producing only oocytes [fem-1 $(h c 17 t s)][9,10]$. Of the predicted genes near mex-3, only two genes, F53G12.6 and F53G12.8, had a fem-3/fem-1 ratio $>5$, indicating sperm-specific expression (Figure 1A). F53G12.8 had already been eliminated as a candidate based on other results (Jeremy Nance, personal communication). We confirmed the sperm-specific expression of F53G12.6 by successful amplification of a PCR product specific from this gene in a fem-3 cDNA library but not in a fem- 1 cDNA library (Figure 1B). Furthermore, a PCR product containing F53G12.6 plus its flanking DNA rescued spe-8(hc50) mutants (Figure 1C). Finally, we identified mutations that altered the F53G12.6 coding sequence in each of the spe-8 mutant strains (Figure 2). Taken together, these data indicate that F53G12.6 is the spe-8 gene.

\section{SPE-8 is a member of a family of $C$. elegans sperm proteins} with non-receptor protein-tyrosine kinase and $\mathrm{SH} 2$ domains. The SPE- 8 protein contains two domains, the first of which is an $\mathrm{SH} 2$ domain (Figure 2B, red line, and Figure 3). SH2 (Src homology 2) is a large family of protein domains that bind particular phosphorylated tyrosine residues on other proteins, and are key regulators of tyrosine phosphorylation signaling cascades [13]. Closely following the $\mathrm{SH} 2$ domain is a non-receptor protein-tyrosine kinase catalytic domain (Figure 2B, blue line, and Figure 3). SPE-8 is a member of the Fes/Fer protein-tyrosine kinase family [14,15]. Alignment of the SPE-8 protein sequence with those of the best matching homologs from a BLASTP search against the nonredundant set of protein databases shows a high degree of conservation and the common presence of the $\mathrm{N}$ terminal $\mathrm{SH} 2$ domain followed by a protein-tyrosine kinase domain, even among distantly related nematodes (Figure 2).

A BLASTP of SPE-8 against C. elegans proteins returns a long list of paralogs (Additional file 2). For the 33 with a match significance $\leq 10^{-30}$, we examined two sources for information on sperm expression. One was the fem-3/fem- 1 ratios from microarray data $[9,10]$, and the second was an analysis of the sperm transcriptome via RNA deep sequencing of sperm purified from him-5 (e1490) males [16]. Twenty-nine of the proteins are upregulated in sperm in at least one of the two studies (Additional file 2). Such a large set of sperm-specific SPE-8 paralogs is not surprising, since spermatogenesis in C. elegans involves an over-abundance of kinases $[9,16]$. All of the 29 sperm-expressed paralogs have the Nterminal SH2 domain followed by the kinase domain. Further, the majority (20) are located on Chromosome IV in a non-random distribution, with some located in two known sperm gene clusters [17] while others lie outside the clusters (Figure 4). The N-terminal $\sim 100$ residues of SPE- 8 define the only region of the sequence that is unique among the sperm-specific paralogs, but the $\mathrm{N}$-terminal domain matches that of all but one of the nematode SPE-8 homologs (Figure 2). Thus, the N-terminal domain likely provides functional specificity for SPE-8. Finally, each of the Caenorhabditis congeners shown in Figure 2B contains a large number of paralogs to its own SPE-8 homolog (data not shown).

We modeled the SPE-8 structure using the I-Tasser Protein Structure and Function Prediction Server [12]. The most significant structural alignments were to the human proteins c-Abl Tyrosine Kinase (Accession: 2FO0) and cSrc Tyrosine Kinase (Accession: 1FMK). While both proteins also included an $\mathrm{SH} 3$ domain very close to the $\mathrm{N}$-Terminus, both proteins possess the $\mathrm{SH} 2$ and kinase domains and have crystal structures available $[18,19]$. The c-Abl Tyrosine Kinase crystal structure was used for modeling SPE-8 with TM-align [11] because it had the most significant structural match $(\mathrm{TM}$-score $=0.866$; Figures 2 and 3). However, SPE-8 has N-terminal and C-terminal ends that were not predicted by c-Abl Tyrosine Kinase structure. We found no significant alignments or predictions for the N-terminal domain (AA's 1-104) considered alone; however, it contains a stretch of seven sequential prolines that may mediate protein-protein interactions [20].

The effects of the various mutations in the spe- 8 gene are illustrated in Figures 2 and 3. All of the missense mutations map to conserved residues (Figure 2B). A single mutation in the SH2 domain was recovered (hc134ts), and it is the only temperature sensitive allele. Mutations found in the predicted minor lobe of the kinase domain, the region responsible for ATP binding, are $h c 53$ and $h c 79$. Mutations found in the predicted major lobe of the kinase domain, the region responsible for recruiting target proteins to the active site, are $h c 40, h c 50$, and $h c 108$. Because $h c 50$ encodes a stop codon, we performed RT-PCR on RNA from $h c 50$ mutants and found that the spe- 8 transcript was present (Additional file 3). Therefore, $h c 50$ eliminates the final 40 amino acid residues from the protein, including a small portion of the kinase domain and the entire C-terminal tail. In many protein kinases, a short $\mathrm{C}$-terminal tail often contains a tyrosine residue, which can be phosphorylated, acting as a cis regulator of the active and inactive state of the protein [20]. The short C-terminus of SPE-8 does not have a tyrosine residue, so it is unclear whether this structure is autoregulatory.

A single mutation, $h c 85$, originally attributed as an allele of spe-8 [4], did not have a mutation in the spe- 8 coding sequence. Our sequencing covered spe- 8 from $\sim 400$ bp 5 ' of start through $\sim 200$ bp 3' of stop, with no alteration from wild-type. To test whether $h c 85$ is actually an allele of spe-8, we performed a complementation test by crossing $h c 85$ hermaphrodites and spe-8(hc50) males. The F1 were fertile, demonstrating that $h c 85$ is not an allele of spe-8. The gene affected by $h c 85$ remains to be determined. 


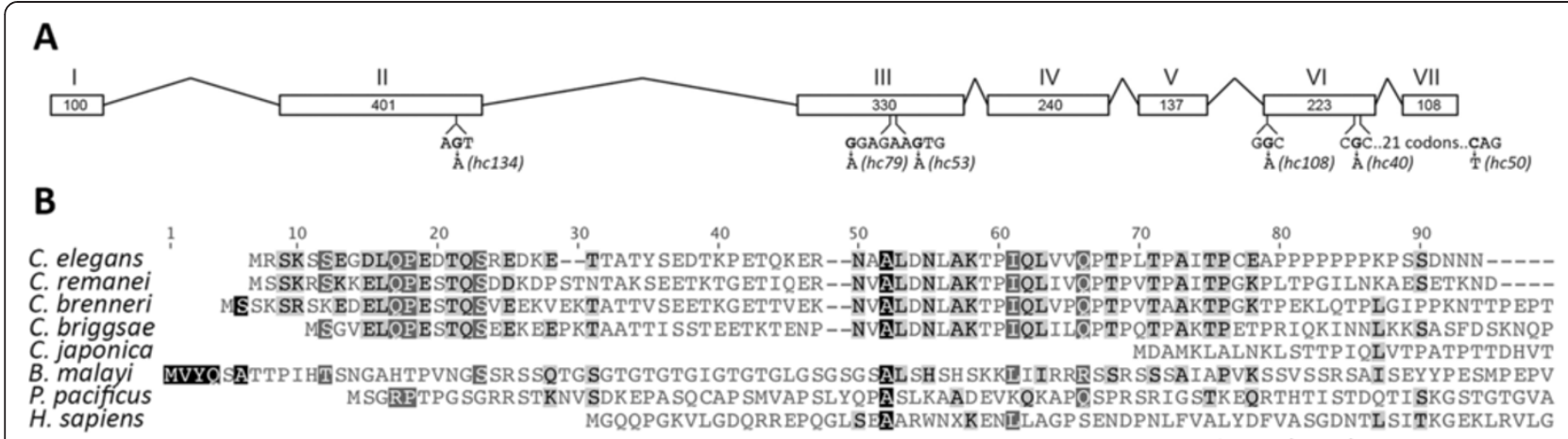

110

120

130

\begin{abstract}
140
\end{abstract}
150

160

$170 f_{180}^{h c 134 t s(S 150 N)}$

190

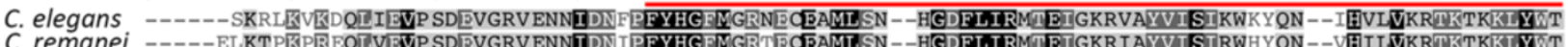

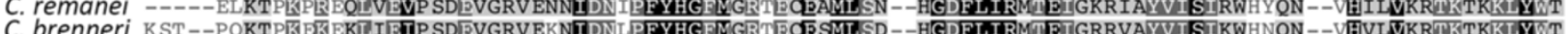

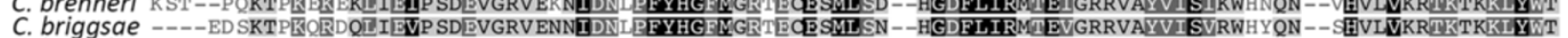

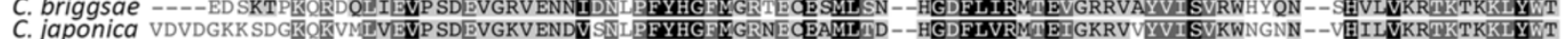

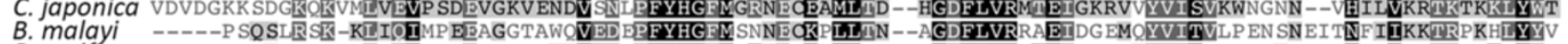

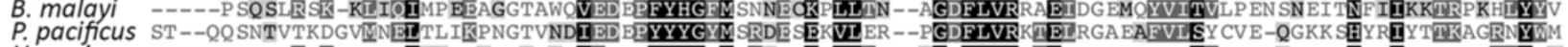

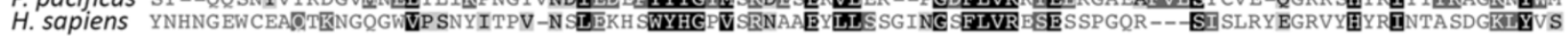
hc79 (G229R) hc53 (V231M)

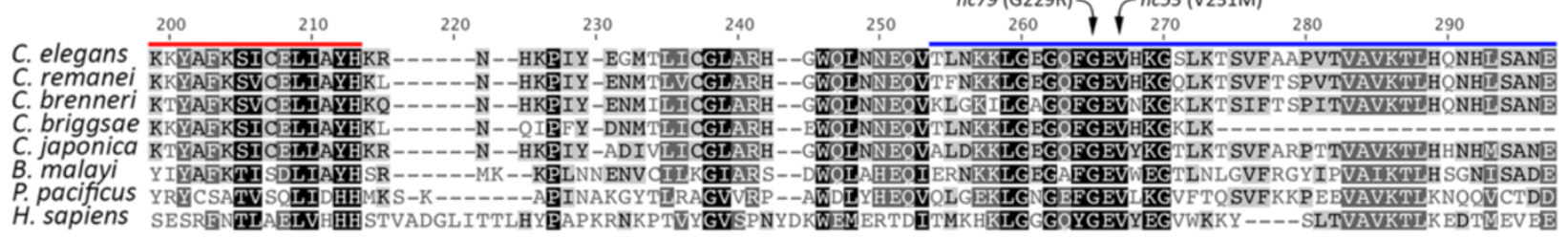

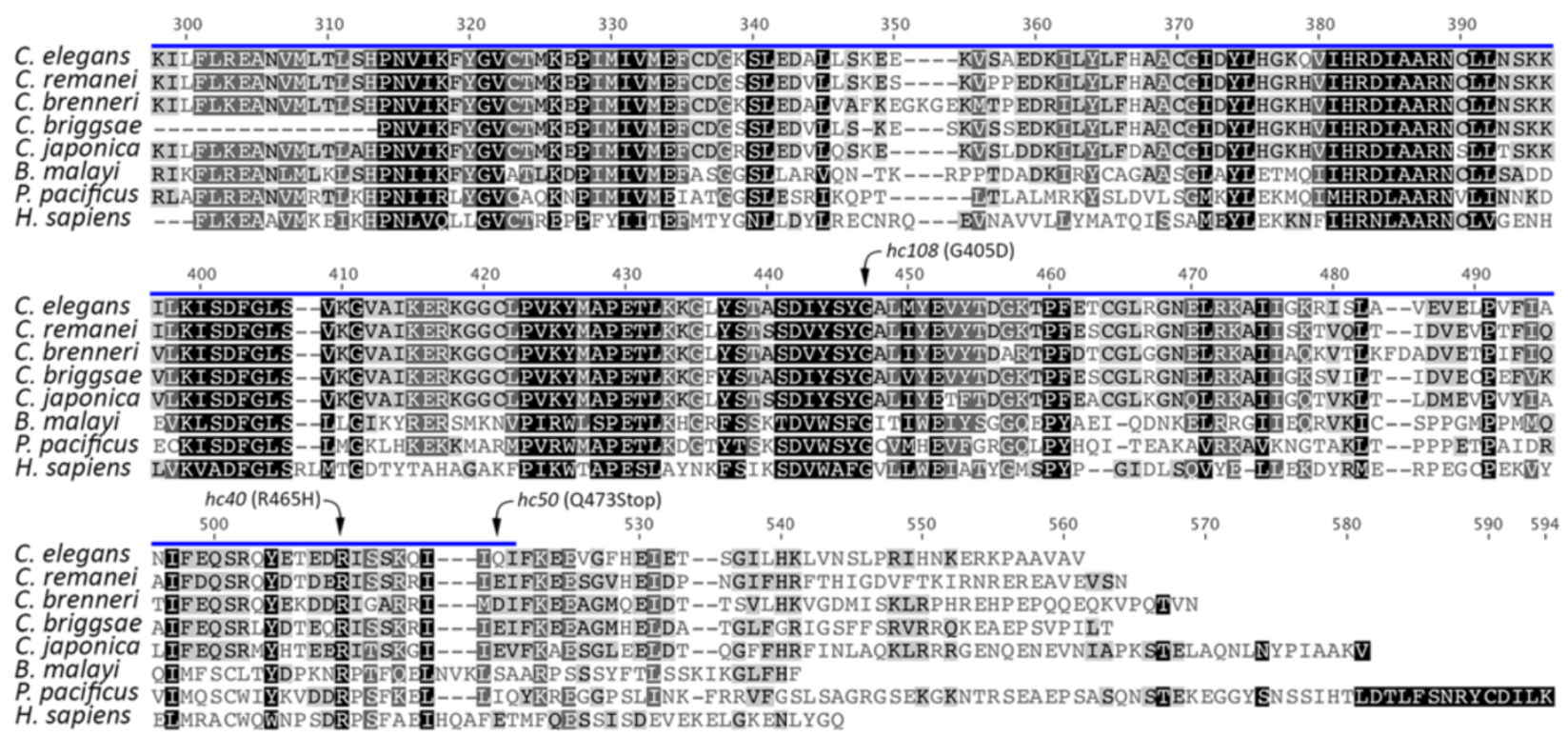

Figure 2 spe-8 exonic structure and sequence alignment of its protein product. The exonic structure shown in A is supported by numerous EST sequences curated by WormBase (www.wormbase.org). The locations of mutations in the spe-8 gene were mapped onto (A) the exon structure and (B) a multiple alignment of SPE-8 from C. elegans and the top BLAST matches from other nematodes. The nucleotide alterations to codons are shown in A, and the amino acid changes are shown in B. Included in the alignment is the human c-Abl Tyrosine Kinase from structural analysis of the protein (Figure 3). The location of the $\mathrm{SH} 2$ domain is indicated by the red line, and the kinase domain is indicated by the blue line.

\section{SPE-8 localization}

Using the Mos-SCI technique [21], we integrated SPE-8:: GFP translational fusions into the ttTi5605 Mos1 transposon insertion on Chromosome II. One fusion had GFP on the N-terminus, and the other had GFP on the
C-terminus. Both showed green fluorescence in the male germline. We crossed worms bearing the two fusions with spe-8(hc50) worms to determine if either reporter fusion rescued the spe- 8 mutant phenotype. For the amino-terminal fusion, we observed 14 sterile worms 


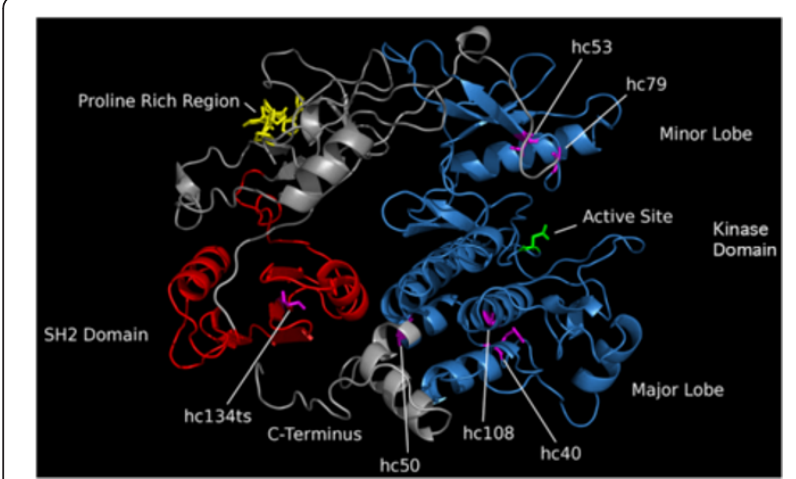

Figure 3 Predicted structural model of SPE-8. The model was predicted by TM-align [11] using iTasser [12], and is based on the crystal structure of the human c-Abl Tyrosine Kinase protein. The highly significant structural predictions for the $\mathrm{SH} 2$ and kinase domains are indicated by red and blue labeling, respectively. The structures of the $\mathrm{N}$ - and C-terminal domains were predicted by iTasser without known protein models and are labeled in gray. The amino acids affected by the spe- 8 mutations are indicated in stick form and labeled purple. Also shown are the predicted kinase active site in green and a proline rich domain in gold.

out of 57 total F2, or 25\%, which is expected from a monohybrid cross with no element present to rescue spe-8. Thus, the amino-terminal fusion did not rescue. However, for the carboxy-terminal fusion, only two of 69 F2 were sterile. This proportion, 2.9\%, is significantly different from the $25 \%$ expected for no rescue $\left(x^{2}=\right.$ 16.41; $\mathrm{P}=0.00005)$, but it is similar to the percent expected if the fusion does rescue, where only $1 / 16(6.2 \%)$ of the worms should be sterile. Thus, the carboxyterminal fusion did rescue the spe-8(hc50) mutation, suggesting that the reporter provides an accurate portrait of SPE-8 localization. Therefore, we used the carboxyterminal fusion to analyze SPE-8 localization.

SPE-8 localizes to the cell membrane during spermatogenesis. All cellular stages of spermatogenesis showed green fluorescence associated with the cell membrane (Figure 5, spe-8::GFP column). Unfortunately, developing sperm cells have autofluorescent internal structures. In comparison, control (non-reporter) developing sperm do not have green fluorescence associated with the cell membrane (Figure 5, N2 column). The primary spermatocytes expressing the reporter appear much more fluorescent even interior to the cell membrane than $\mathrm{N} 2$ controls, suggesting that SPE- 8 may be accumulating in the cytosol and translocating to the membrane at this stage. By the secondary spermatocyte stage, the cytosol appears no more fluorescent than controls, but the plasma membrane is strongly fluorescent as the reporter has taken up residence there. At the budding spermatid stage a strong GFP signal comes from the residual body, where non-essential cellular components are disposed [22]. It is unclear if native SPE- 8 enters the residual body, and an alternative possibility is that some of the fusion has degraded and segregated to the residual body. Finally, the membrane localization of GFP is most pronounced at the spermatid stage, when the cells await the activation signal.

Our results also show that SPE- 8 localization is dependent upon the other spe-8 group proteins. Localization is disrupted in all spe-8 group mutant backgrounds with the possible exception of spe-29 (Figure 5). In spe-12(hc76), spe-19(ok3428), and spe-27 (it132) mutant backgrounds, there is no indication of reporter localization to the membrane. Indeed, the fluorescence seems to concentrate in the residual body, which might be indicative of degradation due to the loss of components of the mature activation complex. In a spe-29(it127) mutant background, reporter localization to the membrane is visible, but it is not as intense as in an otherwise wild-type background, perhaps as a consequence of the likelihood that the spe-29(it127) mutation does not result in a complete loss of function [6].

We also investigated SPE- 8 localization in activated spermatozoa. In naturally activated spermatozoa from both males and hermaphrodites, the membrane localization found in spermatids disperses into the cytosol (Figure 6). Hermaphrodite self sperm activate exclusively through the SPE-8 group pathway signaled by extracellular zinc [2], so entry of SPE-8 into the cytoplasm is a normal consequence of zinc activation through the SPE- 8 group. Male sperm utilize both the SPE-8 pathway and TRY-5 pathway [3]. While activated male sperm showed the same entry of SPE8 into the cytoplasm, it is not clear whether TRY-5 alone releases SPE- 8 from the membrane. What is clear is that TRY-5 activation is completely independent of the SPE-8 group: male spe-8 group mutants are fertile via the TRY-5

Y52D5A.2 C55C3.4 spe-27

Figure 4 The clustered arrangement of the sperm-expressed SPE-8 paralogs on Chromosome IV. The locations of the genes are based upon their nucleotide position, and those above the line are encoded on the positive strand, whereas those below the line are on the negative strand. Known spe genes are also shown. Chromosomal regions highlighted in red indicate known sperm gene clusters [17], and bold font indicates the two sperm genes on Chromosome IV with a sterile knockout phenotype. 


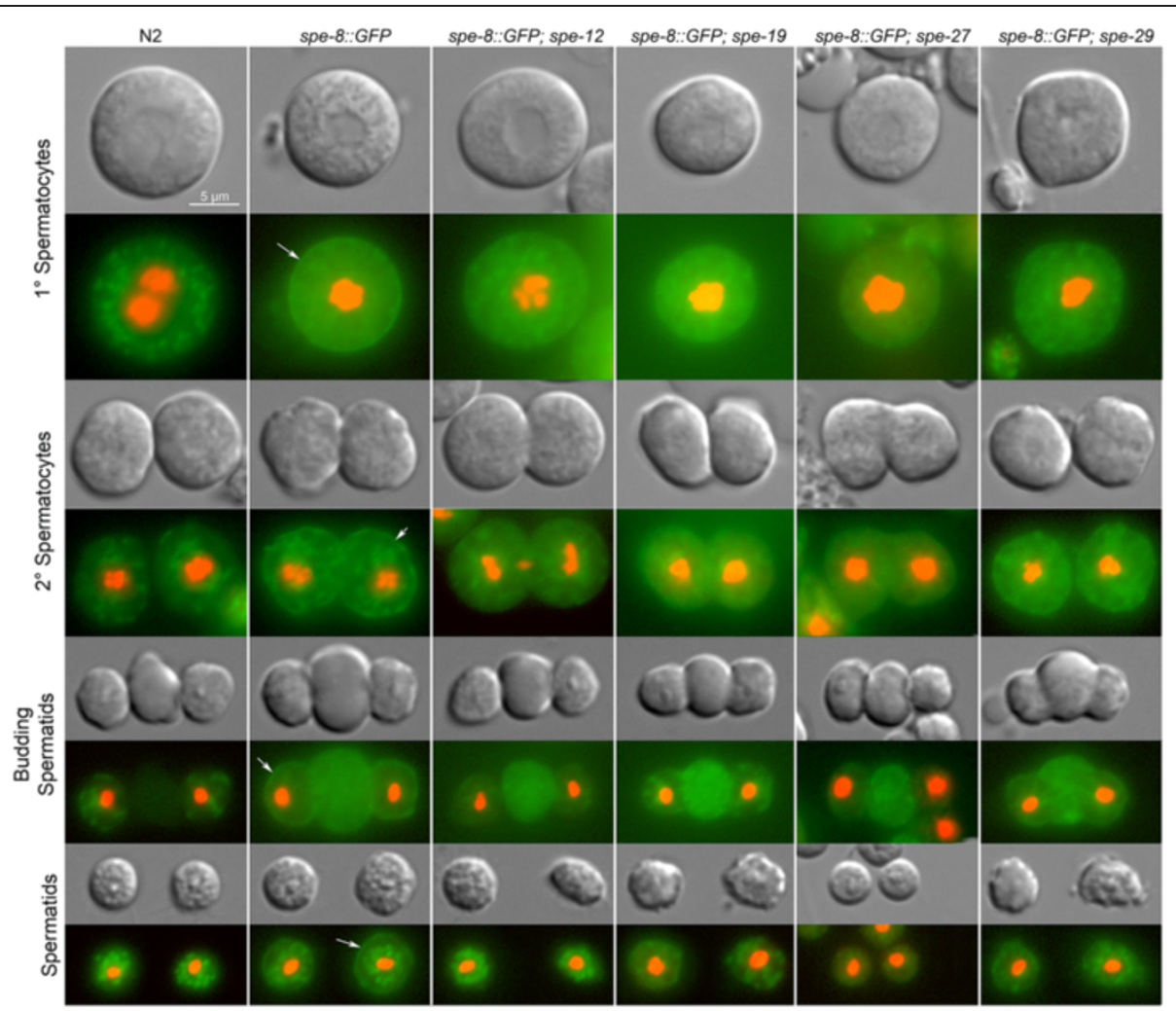

Figure 5 Localization of a spe-8::GFP translational reporter in developing sperm. GFP was fused to the C-terminus of the spe-8 coding sequence and integrated into a Mos1 site on Chromosome II following the MosSCI technique. In the N2 column, cells from the unlabeled N2 strain are included as a negative control to illustrate the non-specific green autofluorescence found in C. elegans developing sperm. The spe-8:: GFP column shows the green fluorescence in cells with the translational reporter. Note the arrows indicating membrane labeling not seen in the $\mathrm{N} 2$ controls. The remaining columns are from worms homozygous for the reporter construct insertion and a mutation in one of the spe-8 group genes. Membrane GFP localization was absent in spe-12(hc76), spe-19(ok3428), and spe-27(it132) mutant backgrounds, but some localization was present in a spe-29(it127) mutant background. Red fluorescence indicates DNA labeling by Hoechst 33342.

pathway, even though our results show that SPE-8 is mislocalized or absent in their sperm. SPE- 8 entry into the cytoplasm also occurs in sperm activated proteolytically via Pronase in vitro (Figure 6). Pronase activation likely acts, at least in part, through the zinc-signaled SPE-8 group proteins, because spe- 8 group mutant spermatids cannot be activated completely to spermatozoa by exposure to Pronase [23]. Interestingly, in vitro activation via triethanolamine (TEA) leaves SPE-8 localized to the membrane (Figure 6), which is not surprising given that TEA bypasses the activation pathway by mimicking a downstream spike in cytosolic $\mathrm{pH}$ [23].

\section{Discussion}

Here, we describe the identification, structure, and function of spe-8, and show that it is localized to the spermatid cell membrane but enters the cytoplasm during activation. spe- 8 was the first of the five genes of the spe-8 group (spe-8, -12,-19, -27, and -29) to be identified by mutation. In contrast to the other four genes, whose sequences reveal little about their activity, the
SPE-8 sequence suggests it belongs to one of the most prominent families of signaling molecules, the protein tyrosine kinases (PTKs) [24]. These proteins route information through cells by phosphorylating specific tyrosine residues on their substrate proteins, thereby altering their substrates' chemical activities and/or binding properties. The Src homology 2 (SH2) domain, a compact domain that recognizes and binds to specific phosphotyrosinecontaining proteins, is a common protein module that is often associated with PTKs and is present in SPE-8. Beyond the strong sequence similarity to these two domains exhibited by SPE-8, the fact that all six of the identified mutations lie in conserved residues of the $\mathrm{SH} 2$ or PTK domain underscores the importance of these domains in SPE-8 function.

PTKs belong to two general classes, the receptor tyrosine kinases (RTKs) and the non-receptor tyrosine kinases (NRTKs) [6]. Whereas RTKs are typically bound to the plasma membrane through their own $\mathrm{N}$-terminal structures, NRTKs may associate with membranes through associations of their N-terminal domains with co-receptor 


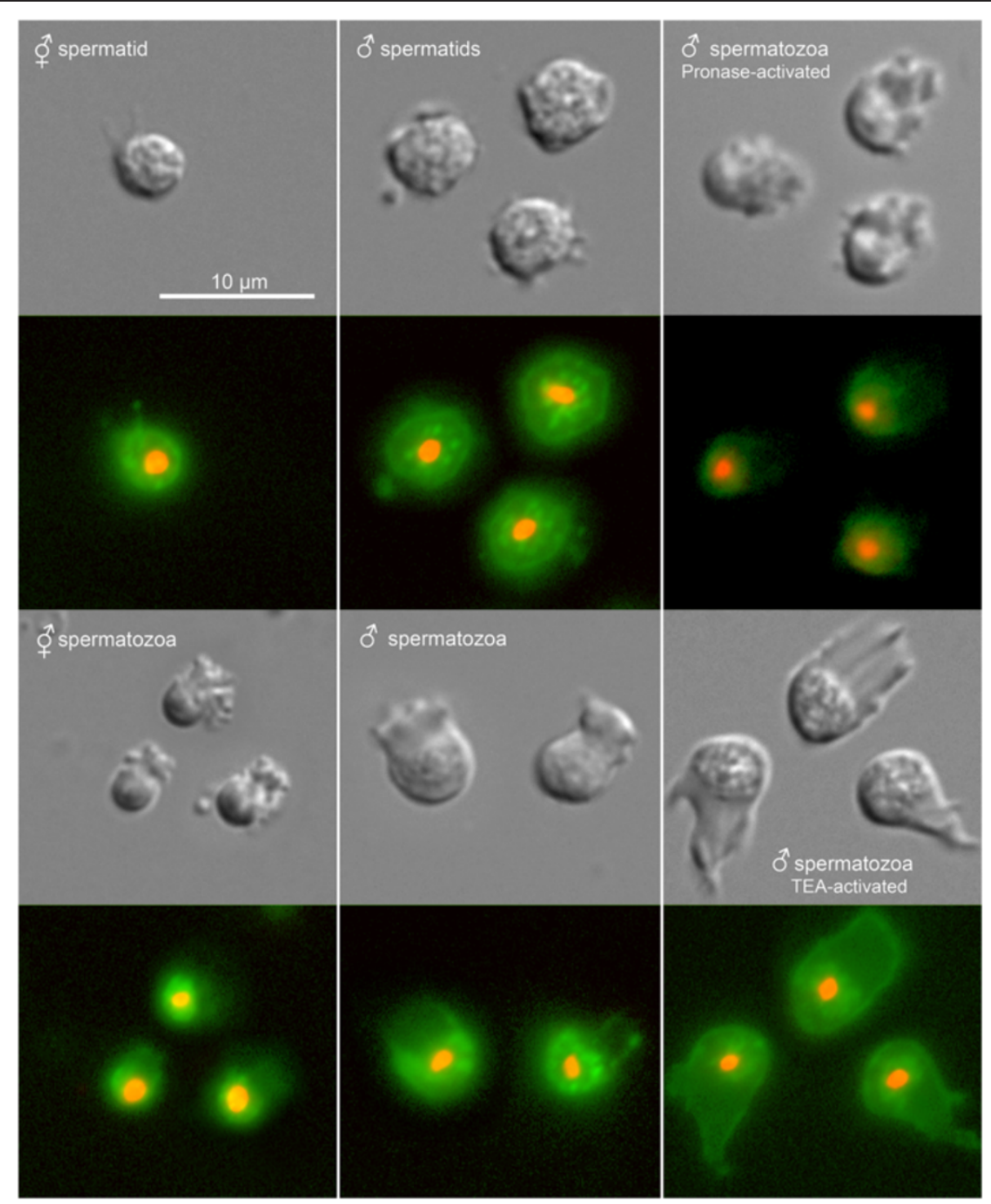

Figure 6 Localization of the spe-8::GFP translational reporter in activated spermatozoa. Spermatids and spermatozoa were isolated from young adult hermaphrodites (first column). The GFP fluorescence has moved to the cytoplasm in the activated spermatozoa. A similar pattern was seen in spermatids and spermatozoa isolated from young adult males (second column). Spermatozoa activated with Pronase also have cytoplasmic GFP localization (third column, top), but when activated with triethanolamine (TEA), the GFP remains at the membrane (third column, bottom).

proteins. Based on overall sequence similarity, SPE-8 is most closely related to the Fer and Fes family of NRTKs [15]. Human Fes has both oncogenic and tumor suppressor roles, depending upon the type of tumor $[14,25]$, and Fer is widely expressed and activated by growth factors, primarily platelet derived growth factor (PDGF) [26]. Fer is involved in many processes including cell proliferation [27,28], cell adhesion [29], cell migration [30], and even oocyte maturation [31]. Fer is also implicated in several cancers [32-34]. Interestingly, a testis-specific alternatively-spliced isoform of Fer, FerT, has a distinct, shorter $\mathrm{N}$-terminus than the full-length Fer protein and participates in acrosome formation through phosphorylation of actin remodeling proteins [35].

Both Fer and Fes have, in addition to their $\mathrm{SH} 2$ and kinase domains, several coiled-coil domains in their N-termini, which appear responsible for activation by oligomerization and subsequent trans-autophosphorylation of these proteins $[18,24]$. NRTKs in general are found to localize in variable fashions either in the cytosol or bound to interior membranes via protein complexes [20]. The SPE-8 Nterminus is unique compared to all other members of the sperm expressed NRTK family in C. elegans. It is unclear if the N-terminus of SPE-8 is responsible for formation of 
dimers or oligomers, or if autophosphorylation occurs. However, we speculate that this structure may be responsible for protein-protein interactions, especially given its proline-rich region, many of which are implicated in protein-protein docking or in binding $\mathrm{SH} 3$ domains $[20,36,37]$. Perhaps the failure of our N-terminal GFP fusion to rescue the spe-8(hc50) mutation reflects the importance of the N-terminus in SPE-8 function. Unlike the unique $\mathrm{N}$-terminus, the SPE-8 C-terminus matches those of some of the family members.

Whatever the function of the $\mathrm{C}$ - and $\mathrm{N}$-terminal domains, it is clear that interactions among the SPE-8 group proteins act to localize or stabilize SPE- 8 at the plasma membrane. Localization was disrupted in mutant backgrounds for spe-12, spe-19, and spe-27, and perhaps for spe-29. It is not surprising that SPE-12 and SPE-19 proteins are required for proper SPE-8 localization because both have transmembrane domains, and SPE-12 has been shown experimentally to reside in the cell membrane [5,7]. However, the requirement of SPE-27 for proper SPE-8 localization is unexpected, given that SPE-27 is predicted to be cytosolic [38]. These results suggest SPE-27 may also be located at the plasma membrane. For SPE-29, the results were not clear. SPE-8 appeared to localize to the membrane in a mutant spe-29 background, but seemingly not as intensely as in a wildtype genomic background.

While SPE-8 associates with the membrane in developing sperm, its localization changes once the cells activate to spermatozoa. The protein appears to leave the membrane and become cytoplasmic in natively activated spermatozoa originating from both males and hermaphrodites. In both cases, the sperm are exposed to extracellular zinc, which acts through the SPE- 8 group proteins to induce activation [2]. We have no method for observing SPE-8::GFP localization in sperm activated only through the TRY-5 pathway. It is not possible to activate sperm in vitro with TRY-5, and spe- 8 group mutant male sperm are activated only by TRY-5, but SPE-8::GFP is mislocalized due to the spe- 8 group mutation. In any case, SPE- 8 is dispensable for TRY-5 activation. Not only does TRY-5 activation occur in spe-8 mutant backgrounds, but SPE-8 is also mislocalized in spe-12, spe-19, and spe-27 mutant backgrounds.

It is tempting to speculate as to the phosphorylation target of SPE-8. One possible target is SPE-6. SPE-6 has a predicted role maintaining the spermatid state by inhibiting activation until the SPE- 8 group signal is transduced into the cell [39]. One hypothesis is that SPE-8 downregulates SPE-6 through tyrosine phosphorylation. SPE-6 protein exists in a perinuclear halo in spermatids (Jackson Peterson and Diane Shakes, personal communication), so the translocation of SPE-8 from the plasmalemma into the cytosol might enable interaction between the two proteins. Indeed, the NetPhos 2.0 Server [40] identifies four of the tyrosine residues in SPE-6 as high probability candidates for phosphorylation (data not shown). Another possible target is a $C$. elegans homolog of MPOP, the major sperm protein polymerization organizing protein identified in Ascaris suum [41,42], although a C. elegans homolog has yet to be found. MPOP is an integral membrane protein distributed throughout the plasmalemma, but it is phosphorylated by a tyrosine kinase only on the leading edge of the pseudopod, where it nucleates MSP assembly to drive pseudopodial motility. However, SPE- 8 is not likely the tyrosine kinase responsible for a putative $C$. elegans MPOP phosphorylation because SPE- 8 appears restricted to the cell body in active spermatozoa (Figure 6).

SPE-8 shares both primary sequence and protein domain architecture similarity with at least 33 other predicted paralogs in C. elegans, 29 of which have been shown to be upregulated during spermatogenesis in at least one of two studies. We include genes identified as sperm-expressed in either study because each study has slight limitations: (i) the microarray studies $[9,10]$ did not include all genes in the genome and may suffer from cross-hybridization, and (ii) the RNA deep sequencing study [16] may miss early acting sperm genes expressed whose transcripts are degraded prior to cellular encapsulation at the primary spermatocyte stage. While the majority of these genes have not received experimental attention, 30 have knockout alleles available. Only four of those knockouts produced a sterile phenotype, with the rest having no phenotype (Additional file 2). RNAi reports suggest that some of the paralogs have somatic function, although even spe- 8 has an RNAi phenotype of hypersensitivity to hypoxia [43]. Whether or not these paralogs have significant function in sperm remains unclear, but the fact that so few have phenotypes for the knockout alleles suggests that they have redundant function. The fact that so many kinases appear upregulated in sperm indicates that protein phosphorylation is a prominent regulatory mechanism during spermatogenesis. Such a conclusion is not surprising since (i) posttranslational modification is the major regulatory mechanism available, owing to the cessation of gene expression at the spermatid stage, and (ii) protein phosphorylation is rapid, befitting the swift morphological transformations that take place during spermiogenesis.

Extracellular zinc is the signal for sperm activation transduced through the SPE- 8 complex [2]. The zinc signal is transmitted across the membrane to SPE- 8 tethered just inside the membrane. It is unclear how reception of the zinc signal releases SPE-8 from the membrane. Zinc is an important signaling molecule in numerous cell types. For instance, the activity of most cell types in the mammalian immune system is regulated by zinc [44]. Zinc alters sperm activity across many species, but its effect can be either 
positive or negative, depending upon the species (reviewed in [2]). Perhaps a more relevant example is the activation of p70 S6 kinase by extracellular zinc signaling in the progression of the cell cycle, with the possible involvement of intermediate NRTKs [45]. While the identities of all intermediate factors and their specific interactions are not known, extracellular zinc induced signaling involving kinases is widespread, and our work on SPE-8 helps in understanding how spatial localization is involved in the signaling events.

\section{Conclusions}

The spermatid activation signal initiated by extracellular zinc is transduced into the cell via the SPE- 8 group proteins. Of the five proteins known to compose this pathway, only SPE-8 has a predicted functional domain, that being a non-receptor protein-tyrosine kinase. Three have predicted membrane domains (SPE-12, SPE-19, and SPE-29), and SPE-27 has no predicted domains. The association of the SPE-8 group proteins has been unclear. Our results suggest that the SPE- 8 group proteins form a functional complex localized at the plasma membrane, and that SPE-8 is correctly positioned only when all members of the SPE- 8 group are present, with the possible exception of SPE-29. Further, SPE-8 is released from the membrane when the activation signal is transduced into the spermatid. The identity of its phosphorylation target(s) is not yet clear, nor is whether SPE-8 kinase activity is up- or down-regulated by the activation signal. SPE-8 is also a member of a large family of sperm-expressed non-receptor protein tyrosine kinases in $C$. elegans, suggesting that the rapid changes associated with spermatogenesis - and spermatid activation in particular - are brought about, at least in part, through changes in phosphorylation.

\section{Methods}

\section{Strains and general nematode methods}

Worm strains were cultured by standard methods [46]. Some strains were provided by the Caenorhabditis Genetics Center (CGC), which is funded by NIH Office of Research Infrastructure Programs (P40 OD010440). Others were either generated during the present work or were graciously provided by Samuel Ward and Steven W. L'Hernault. The following strains harbored spe-8 group gene mutations: BA785: spe-8(hc40) I, BA784: spe8(hc50) I, BA786: spe-8(hc53) I, BA788: spe-8(hc79) I, BA801: spe-8(hc85) I (although we show here that $h c 85$ is not an allele of spe-8), BA787: spe-8(hc108) I, BA797: spe-8(hc134ts) I, VC2971: spe-19(ok3428)/unc-51(e369) rol-9(sc148) V, BA963: spe-27(it132ts) IV, BA959: spe-29 (it127) dpy-20(e1282) IV, BA783: spe-12(hc76) I. Translational GFP reporters were integrated into the ttTi5605 Mos1 insertion site in the strain EG4322: ttTi5605 II; unc-119(ed9) III. Sperm were activated in vitro using Pronase and triethanolamine following standard protocols [23].

\section{Differential PCR analysis of spe-8}

We used PCR to assess sperm-specific expression of spe-8. PCR was conducted on cDNA libraries constructed from either fem-1(hc17ts) or fem-3(q23ts) mutants (kindly provided by Harold Smith). Three different products were amplified using different primer pairs: (i) a spe- 8 product (primers F53G12.6Lb and F53G12.6 L3'), (ii) a spe-12 product as a sperm upregulated positive control [7], and (iii) a product from F2SH8.1, which is expressed somatically (Jeremy Nance, personal communication). PCR primer sequences are listed in Additional file 1. PCR reaction conditions and cycling followed standard protocols [47].

\section{Microinjection transformation rescue}

We amplified a 4,273 bp fragment from wild-type worms containing the entire F53G12.6 gene, including 919 base pairs $5^{\prime}$ of the start codon, and 592 base pairs 3' from the stop codon. We used Phusion High Fidelity DNA Polymerase (Thermo Scientific) for amplification following manufacturer's protocols. For transformation rescue, a mixture containing $10 \mathrm{ng} / \mu \mathrm{l}$ of this PCR fragment, along with $100 \mathrm{ng} / \mu \mathrm{l}$ of the pCFJ104 plasmid containing the Pmyo-3::mCherry::unc-54 transcriptional fusion to track transformed progeny. The DNA mix was injected into young adult spe-8(hc50) mutant hermaphrodites that were then paired with males of the same mutant genotype. F1 and F2 hermaphrodite transformants were scored for self-fertility at $25^{\circ} \mathrm{C}$ using non-transformant sibs as controls.

\section{Sequencing the spe- 8 mutants}

To sequence the spe- 8 mutant alleles, the spe- 8 genomic region from the mutants was amplified by PCR either in two $\sim 1,300$ bp fragments (for mutations hc40, hc50, $h c 53, h c 79, h c 108$, and hc134ts), or in a single large product corresponding to the rescuing fragment (for hc85). Phusion High Fidelity DNA Polymerase was used in amplification, and the fragments were sequenced by the Sanger method using the PCR primers and primers internal to the two fragments. All primer sequences are listed in Additional file 1.

\section{RT-PCR}

RNA was extracted from a mixed age population of spe8 (hc50) worms. The worms were rinsed 4 times with M9 buffer, disrupted with a homogenizer in lysis buffer from the GeneJET ${ }^{\mathrm{TM}}$ RNA purification kit (Thermo Scientific), and the RNA was extracted following the manufacturer's protocols. The RNA samples were treated with RQ1 DNase (Promega), and reverse transcription was performed with 
Maxima $^{\text {тм }}$ Reverse Transcriptase (Thermo Scientific) per manufacturer's instructions with oligo (dT). We then multiplexed spe-8 specific primers (F53G12.6-F5 and F53G12.6-R5; see Additional file 1) with primers specific to act-2, the $C$. elegans ortholog of $\beta$-actin (see Additional file 1 for primer sequences) for amplification using Phusion High Fidelity DNA Polymerase (Thermo Scientific) per manufacturer's protocols.

\section{SPE-8 localization}

Two translational GFP fusions with spe- 8 were constructed following the Mos-SCI technique [21]. Both constructs included 838 bp of the sequence upstream of the start codon and 1039 bp downstream of the stop codon. The GFP coding sequence from plasmid pPD95.75 (Fire Lab C. elegans vector kit obtained from Addgene) was inserted either at the 5 ' or the 3' end of the genomic spe- 8 sequence. The stop codon was eliminated from (i) GFP for the aminoterminal construct, or (ii) spe- 8 for the carboxy-terminal construct. The various segments of the construct were amplified by PCR using Phusion High Fidelity DNA Polymerase (Thermo Scientific), and they were combined following the PCR fusion technique described by Hobert [48]. Primers used in the construction are listed in Additional file 1. The full-length constructs were cloned into the vector pCFJ151, which targets the ttTi5605 Mos1 transposon insertion on Chromosome II for homologous recombination. Following the Mos-SCI protocol [21], we recovered a homozygous integrated copy of each construct. The integrated reporters were crossed with spe-8(hc50) to determine if the reporters rescued spe- 8 mutant sterility. The C-terminal reporter (zqIs8[spe-8::GFP]) rescued spe-8 mutant sterility (see Results) and was used in localization studies. We crossed the zqIs8[spe-8::GFP] translational reporter with mutations in the various spe- 8 group genes to obtain strains that were homozygous for both $z q I s 8$ and for the spe- 8 group mutations. Microscopy was performed with a Nikon Eclipse Ti-S inverted microscope fitted for Differential Interference Contrast microscopy and epifluorescence. Localization was examined in the following strains: ZQ125: zqIs8 II; unc-119(ed3) III, ZQ126: spe-12(hc76) I; zqIs8 II, ZQ127: zqIs8 II; spe-29(it127) dpy-20(e1282ts) IV, ZQ128: zqIs8 II; spe-19(eb52) V, and ZQ137: zqIs8 II; spe-27 (it132ts) IV.

\section{Additional files}

Additional file 1: Primers used in PCR amplification and sequencing.

Additional file 2: $C$. elegans SPE-8 paralogs. SPE-8 and its paralogs with a match significance $\leq 10^{-30}$. The chromosome and start position are given for each paralog, as is the RPKM (Reads Per Kilobase of exon model per Million mapped reads) from RNA-seq for sperm gene identification [16]. The presence of a value in RPKM indicates upregulation in sperm. fem-3/fem- 1 expression ratio, where larger ratios indicate upregulation in sperm [9]. If available, the identity of a knockout allele, and its phenotype are listed. Phenotype data were taken from www.wormbase.org, version WS240.

Additional file 3: RT-PCR for spe-8 in a spe-8(hc50) mutant. RNA was extracted and DNase treated and then subjected to RT-PCR for both a fragment of the spe- 8 coding sequence and for a fragment of the gene act- 2 , a homolog of $\beta$-actin. The primers for spe- 8 and act- 2 are listed in Additional file 1. Both primer sets cross introns, and the resulting products would be larger if amplified from genomic DNA (act-2: 1050 bp; spe-8: 565 bp).

\section{Competing interests}

The author(s) declare that they have no competing interests.

\section{Authors' contributions}

PJM conceived of the spe- 8 identification study and its design, carried out the discovery of the spe- 8 coding sequence, and aided in the sequencing of the mutants, discovery of paralogs, and drafting of the manuscript. JNC participated in the sequencing of the mutants and drafting of the manuscript. UN contributed to the construction of the reporter strains, RT-PCR, and drafting the manuscript. NGS modeled the SPE-8 protein and participated in drafting the manuscript. CWL conceived and designed the localization studies, aided in project design, created the reporter constructs, performed the microscopy, and contributed to drafting the manuscript. All authors read and approved the final manuscript.

\section{Acknowledgements}

We thank Andrew Singson for the continued urging to complete this project so that he could cite something other than WormBase for spe-8. Harold Smith, Jeremy Nance, and Samuel Ward provided helpful advice and discussion. Harold Smith also kindly provided the fem-1 and fem-3 cDNA libraries.

\section{Author details}

'Department of Molecular and Cellular Biology, University of Arizona, 1007 Lowell St., Tucson, AZ 85721, USA. ²Department of Molecular, Cellular and Developmental Biology, Currently, University of Colorado Boulder, 347 UCB, Boulder, CO 80309, USA. '3epartment of Biological Science, California State Polytechnic University, 3801 W. Temple Ave, Pomona, CA 91768, USA.

Received: 9 April 2014 Accepted: 3 July 2014

Published: 14 July 2014

\section{References}

1. Ellis RE, Stanfield GM: The regulation of spermatogenesis and sperm function in nematodes. Semin Cell Dev Biol 2014, 29C:17-30.

2. Liu Z, Chen L, Shang Y, Huang P, Miao L: The micronutrient element zinc modulates sperm activation through the SPE-8 pathway in Caenorhabditis elegans. Development 2013, 140(10):2103-2107.

3. Smith JR, Stanfield GM: TRY-5 is a sperm-activating protease in Caenorhabditis elegans seminal fluid. PLoS Genet 2011, 7(11):e1002375.

4. L'Hernault SW, Shakes DC, Ward S: Developmental genetics of chromosome I spermatogenesis-defective mutants in the nematode Caenorhabditis elegans. Genetics 1988, 120(2):435-452.

5. Geldziler B, Chatterjee I, Singson A: The genetic and molecular analysis of spe-19, a gene required for sperm activation in Caenorhabditis elegans. Dev Biol 2005, 283(2):424-436.

6. Nance J, Davis EB, Ward S: spe-29 encodes a small predicted membrane protein required for the initiation of sperm activation in Caenorhabditis elegans. Genetics 2000, 156(4):1623-1633.

7. Nance J, Minniti AN, Sadler C, Ward S: spe-12 encodes a sperm cell surface protein that promotes spermiogenesis in Caenorhabditis elegans. Genetics 1999, 152(1):209-220.

8. Draper BW, Mello CC, Bowerman B, Hardin J, Priess JR: MEX-3 is a KH domain protein that regulates blastomere identity in early $C$. elegans embryos. Cell 1996, 87(2):205-216.

9. Reinke V, Smith HE, Nance J, Wang J, Van Doren C, Begley R, Jones SJ, Davis EB, Scherer S, Ward S, Kim SK: A global profile of germline gene expression in C. elegans. Mol Cell 2000, 6(3):605-616. 
10. Reinke V, Gil IS, Ward S, Kazmer K: Genome-wide germline-enriched and sex-biased expression profiles in Caenorhabditis elegans. Development 2004, 131(2):311-323.

11. Zhang Y, Skolnick J: TM-align: a protein structure alignment algorithm based on the TM-score. Nucleic Acids Res 2005, 33(7):2302-2309.

12. Roy A, Kucukural A, Zhang Y: I-TASSER: a unified platform for automated protein structure and function prediction. Nat Protoc 2010, 5(4):725-738.

13. Sawyer TK: Src homology-2 domains: structure, mechanisms, and drug discovery. Biopolymers 1998, 47(3):243-261.

14. Craig AW: FES/FER kinase signaling in hematopoietic cells and leukemias. Front Biosci 2012, 17:861-875.

15. Smithgall TE, Rogers JA, Peters KL, Li J, Briggs SD, Lionberger JM, Cheng $H_{\text {, }}$ Shibata A, Scholtz B, Schreiner S, Dunham N: The c-Fes family of proteintyrosine kinases. Crit Rev Oncog 1998, 9(1):43-62.

16. Ma X, Zhu Y, Li C, Xue P, Zhao Y, Chen S, Yang F, Miao L: Characterisation of Caenorhabditis elegans sperm transcriptome and proteome. BMC Genomics 2014, 15:168.

17. Miller MA, Cutter AD, Yamamoto I, Ward S, Greenstein D, Dunham N: Clustered organization of reproductive genes in the $\mathrm{C}$ elegans genome. Curr Biol 2004, 14(14):1284-1290.

18. Xu W, Harrison SC, Eck MJ: Three-dimensional structure of the tyrosine kinase c-Src. Nature 1997, 385(6617):595-602.

19. Nagar B, Hantschel O, Seeliger M, Davies JM, Weis WI, Superti-Furga G, Kuriyan J: Organization of the $\mathrm{SH} 3-\mathrm{SH} 2$ unit in active and inactive forms of the c-Abl tyrosine kinase. Mol Cell 2006, 21(6):787-798.

20. Birge RB, Knudsen BS, Besser D, Hanafusa H: SH2 and SH3-containing adaptor proteins: redundant or independent mediators of intracellular signal transduction. Genes Cells 1996, 1(7):595-613.

21. Frokjaer-Jensen C, Davis MW, Hopkins CE, Newman BJ, Thummel JM, Olesen SP, Grunnet M, Jorgensen EM: Single-copy insertion of transgenes in Caenorhabditis elegans. Nat Genet 2008, 40(11):1375-1383.

22. Ward S: The asymmetric localization of gene products during the development of Caenorhabditis elegans spermatozoa. In Gametogenesis and the Early Embryo. Edited by Gall J. New York: A.R. Liss; 1986:55-75.

23. Ward S, Hogan E, Nelson GA: The initiation of spermiogenesis in the nematode Caenorhabditis elegans. Dev Biol 1983, 98(1):70-79.

24. Hubbard SR, Till JH: Protein tyrosine kinase structure and function. Annu Rev Biochem 2000, 69:373-398.

25. Zhang S, Chitu V, Stanley ER, Elliott BE, Greer PA: Fes tyrosine kinase expression in the tumor niche correlates with enhanced tumor growth, angiogenesis, circulating tumor cells, metastasis, and infiltrating macrophages. Cancer Res 2011, 71(4):1465-1473.

26. Kim L, Wong TW: The cytoplasmic tyrosine kinase FER is associated with the catenin-like substrate pp 120 and is activated by growth factors. Mol Cell Biol 1995, 15(8):4553-4561.

27. Allard P, Zoubeidi A, Nguyen LT, Tessier S, Tanguay S, Chevrette M, Aprikian A, Chevalier S: Links between Fer tyrosine kinase expression levels and prostate cell proliferation. Mol Cell Endocrinol 2000, 159(1-2):63-77.

28. Zoubeidi A, Rocha J, Zouanat FZ, Hamel L, Scarlata E, Aprikian AG, Chevalier $S$ : The Fer tyrosine kinase cooperates with interleukin- 6 to activate signal transducer and activator of transcription 3 and promote human prostate cancer cell growth. Mol Cancer Res 2009, 7(1):142-155.

29. Yoneyama T, Angata K, Bao X, Courtneidge S, Chanda SK, Fukuda M: Fer kinase regulates cell migration through alpha-dystroglycan glycosylation. Mol Biol Cell 2012, 23(5):771-780.

30. Itoh T, Hasegawa J, Tsujita K, Kanaho Y, Takenawa T: The tyrosine kinase Fer is a downstream target of the PLD-PA pathway that regulates cell migration. Sci Signal 2009, 2(87):ra52.

31. McGinnis LK, Carroll DJ, Kinsey WH: Protein tyrosine kinase signaling during oocyte maturation and fertilization. Mol Reprod Dev 2011, 78(10-11):831-845

32. Li H, Ren Z, Kang $X$, Zhang L, Li X, Wang Y, Xue T, Shen Y, Liu Y: Identification of tyrosine-phosphorylated proteins associated with metastasis and functional analysis of FER in human hepatocellular carcinoma cells. BMC Cancer 2009, 9:366.

33. Ivanova IA, Vermeulen JF, Ercan C, Houthuijzen JM, Saig FA, Vlug EJ, van der Wall E, van Diest PJ, Vooijs M, Derksen PW: FER kinase promotes breast cancer metastasis by regulating alpha6- and beta1-integrin-dependent cell adhesion and anoikis resistance. Oncogene 2013, 32(50):5582-5592.

34. Rocha J, Zouanat FZ, Zoubeidi A, Hamel L, Benidir T, Scarlata E, Brimo F, Aprikian A, Chevalier S: The Fer tyrosine kinase acts as a downstream interleukin- 6 effector of androgen receptor activation in prostate cancer. Mol Cell Endocrinol 2013, 381(1-2):140-149.

35. Kierszenbaum AL, Rivkin E, Tres LL: Expression of Fer testis (FerT) tyrosine kinase transcript variants and distribution sites of FerT during the development of the acrosome-acroplaxome-manchette complex in rat spermatids. Dev Dyn 2008, 237(12):3882-3891.

36. Bernacchi S, Mercenne G, Tournaire C, Marquet R, Paillart JC: Importance of the proline-rich multimerization domain on the oligomerization and nucleic acid binding properties of HIV-1 Vif. Nucleic Acids Res 2011 39(6):2404-2415.

37. Ren $\mathrm{X}$, Hurley JH: Proline-rich regions and motifs in trafficking: from ESCRT interaction to viral exploitation. Traffic 2011, 12(10):1282-1290

38. Minniti AN, Sadler C, Ward S: Genetic and molecular analysis of spe-27, a gene required for spermiogenesis in Caenorhabditis elegans hermaphrodites. Genetics 1996, 143:213-223.

39. Muhlrad PJ, Ward S: Spermiogenesis Initiation in Caenorhabditis elegans Involves a Casein Kinase 1 Encoded by the spe-6 Gene. Genetics 2002, 161(1):143-155.

40. Blom N, Gammeltoft S, Brunak S: Sequence and structure-based prediction of eukaryotic protein phosphorylation sites. J Mol Biol 1999, 294(5):1351-1362.

41. LeClaire LL 3rd, Stewart M, Roberts TM: A 48 kDa integral membrane phosphoprotein orchestrates the cytoskeletal dynamics that generate amoeboid cell motility in Ascaris sperm. J Cell Sci 2003, 116(Pt 13):2655-2663.

42. Yi K, Buttery SM, Stewart M, Roberts TM: A Ser/Thr kinase required for membrane-associated assembly of the major sperm protein motility apparatus in the amoeboid sperm of Ascaris. Mol Biol Cell 2007, 18(5):1816-1825.

43. Gort EH, van Haaften G, Verlaan I, Groot AJ, Plasterk RH, Shvarts A, Suijkerbuijk KP, van Laar T, van der Wall E, Raman V, Van Diest PJ, Tijsterman M, Vooijs M: The TWIST1 oncogene is a direct target of hypoxia-inducible factor-2alpha. Oncogene 2008, 27(11):1501-1510.

44. Haase $H$, Rink L: Functional significance of zinc-related signaling pathways in immune cells. Annu Rev Nutr 2009, 29:133-152.

45. Kim S, Jung Y, Kim D, Koh H, Chung J: Extracellular zinc activates p70 S6 kinase through the phosphatidylinositol 3-kinase signaling pathway. J Biol Chem 2000, 275(34):25979-25984.

46. Brenner S: The genetics of Caenorhabditis elegans. Genetics 1974, 77:71-94.

47. Liau WS, Nasri U, Elmatari D, Rothman J, LaMunyon CW: Premature sperm activation and defective spermatogenesis caused by loss of spe-46 function in Caenorhabditis elegans. PloS One 2013, 8(3):e57266.

48. Hobert O: PCR fusion-based approach to create reporter gene constructs for expression analysis in transgenic C. elegans. Biotechniques 2002, 32(4):728-730.

doi:10.1186/1471-2156-15-83

Cite this article as: Muhlrad et al:: SPE-8, a protein-tyrosine kinase, localizes to the spermatid cell membrane through interaction with other members of the SPE- 8 group spermatid activation signaling pathway in $C$. elegans. BMC Genetics 2014 15:83.

\section{Submit your next manuscript to BioMed Central and take full advantage of:}

- Convenient online submission

- Thorough peer review

- No space constraints or color figure charges

- Immediate publication on acceptance

- Inclusion in PubMed, CAS, Scopus and Google Scholar

- Research which is freely available for redistribution 